\title{
ASSESSING ACCEPTABILITY OF PARENTS/GUARDIANS OF ADOLESCENTS TOWARDS INTRODUCTION OF SEX AND REPRODUCTIVE HEALTH EDUCATION IN SCHOOLS AT KINONDONI MUNICIPAL IN DAR ES SALAAM CITY
}

Abstract:

\author{
Lumuli Mbonile ${ }^{1}$ and Edmund J. Kayombo ${ }^{2}$
}

\begin{abstract}
Objectives: To assess acceptability of parents/guardians of adolescents towards the introduction of sex and reproductive health education in the community and schools.

Methods: A multi-stage random sampling technique was used to get 150 participants for this study. A structured questionnaire was used to interview the sampled participants and was supplemented with guided focus group discussion in Kinondoni Municipality of Dar es Salaam, Tanzania.

Results: The analysis of the findings shows that there is a mixed feeling on the introduction of sex and reproductive health education in schools. Participants strongly supported that they should talk with their adolescents about sexuality and reproductive health (88.6\%) but their culture prohibits them from doing so (76.7\%). Also supported that condoms could protect against HIV/AIDS and sexually transmitted infections $(82 \%)$, but strongly opposed the use of condoms to their adolescents because it would encourage promiscuity $(\mathbf{7 8 \%})$. When the data were analysed by faith of the religions of the participants, $64 \%$ were in favour of introducing sex education and reproductive health, but were opposed to use of condoms to their adolescents. All participants were against vijiweni, which were recreation centres for the youths because they taught bad manners to their adolescents. The preferred source of information about sex education and reproductive health should be from the parents/guardians $(86 \%)$, religious leaders $(70 \%)$, media $(62 \%)$, health workers $(61 \%)$ and school teachers $(59 \%)$.

Conclusion: All in all the will of introduction of sex education and reproductive health in the community is there but the approach need to be worked out carefully by taking into account of the cultural and religious factors. Parents/guardians, religious leaders and traditional charismatic leaders should take part in designing the programme and even being involved in teaching it. The other option is to lump together sex education and reproductive health education in science especially in biology which is already in place in Tanzania education programmes.
\end{abstract}

Key words: Tanzania, Sex education, Reproductive health, Parents/guardians, Initiation to adulthood

\section{Introduction}

Introduction of sex and reproductive health education in schools and families aims to fill in the gaps of traditional socio-structures that were involved in the initiation of adolescents to adulthood in most African ethnic groups including Tanzania (1-3). Sex and reproductive health education focus on sexual health and well being in a person's sexual life-feeling comfortable and confident about sex and sexuality, being able to avoid sexually transmitted diseases (STDs), and unwanted pregnancies, ensuring fertility and safe pregnancies, and protecting the health of infants (4). This can be achieved by planned preparation of adolescents in the transition period to adulthood in the family or in schools or in the community; or as was done in the past during the initiation period to adulthood. It becomes a tragedy when children secretly learn about their bodies and sexuality from encyclopaedia or on the street (5).

Pre-colonials and even after independence in African communities south of the Sahara there were social structures that were involved in the initiation of youths and played an important role in preparing adolescents to adulthood $(1,2,6,7)$. For boys age, the initiation was at 10 years, whereas for girls were after first menstrual period $(1,6,7)$. At the general level, adolescents were taught matters related to socio-economic activities they were expected to play in their respective communities including useful herbal plants in the daily health problems (8-10). Sexuality and reproductive health were treated separately according to sex, for the girls were elderly women and for boys were elderly men $(1,6,7)$.

(to: Edmund E. Kayombo, P.O. Box 65586, Dar es Salaam, Tanzania, Email: ekayombo@yahoo.co.uk

${ }^{1}$ School of Medicine, Muhimbili University of Health \& Allied Sciences (MUHAS), Dar es Salaam, Tanzania; ${ }^{2}$ Institue of Traditional Medicine, MUHAS
The training of sex and reproductive health education was regarded as sensitive and the opposite sex were restricted to enter in the area of training. The trainees on the other hand were strictly forbidden not to tell anybody, besides themselves and the trainers, of what they have been taught or seen. Going against it was regarded as transgressing the norms of that ethnic group and sometimes the individual may be severely punished $(1,2)$.

There was special emphasis on reproductive health and hygiene to girls $(1,2,6)$. Virginity was valued more to girls than to boys. Pregnancies were rare to unmarried young girls; and if it happens the girl and sometimes the whole family was stigmatised and rebuked in some ethnic groups $(1,6,7)$. Furthermore, everybody was responsible for the upbringing of the adolescents to make them behave according to the norms of the community $(1,2,6,7)$. In Tanzania, for example, in some ethnic groups there were special old people who were accepted by the communities for the initiation on ceremonies of adolescents to adulthood according to sex, for the girls were elderly women referred as kungwi and for boys were elderly men referred as ngaliba. The ngaliba and kungwi are also referred as somo (1-3,6-7). In some ethnic groups the grand parents and aunts were the ones who were responsible for the initiation of adolescents to adulthood $(1,3,6)$.

The traditional socio-cultural structures that were used for communicating with adolescents in the past are declining in recent years with modernisation, introduction of various source of communication and urban type of life. All these are actors as the instruments of behavioural change $(3,11,12)$. Many people have changed their perception of traditional practices of bringing up children and take them as archaic because they are not conforming to the current changes(3). With the increasing 
modernisation these socio-structures that were involved in the initiation to adulthood have been eroded, and where they tend to exist the initiation process is partially done to adolescents $(3,13)$; and hence the adolescents are at the cross-roads as to who to consult on issues of sexuality and related health issues. The impact of these is that most of the adolescents rely on the information in the newspapers and television $(3,4,13)$. This in turn has led adolescents to be involved in unsafe sex leading to unplanned pregnancy, illegal abortion, HIV/AIDS and sexually transmitted infections $(3,4,13-15)$. Because these reproductive health problems including HIV infections are highly stigmatised, adolescents do not know where to seek health care when infected.

Increasing rate of unplanned pregnancy, illegal abortion, HIV/AIDS and sexual transmitted infections among the adolescents $(4,15,16)$ has made policy makers to look for an alternative structure to prevent the increasing rate of the mentioned health problems. One of the strategies is by introduction of sex and reproductive health education with the aim of promoting a broader definition of safer sex which is pleasurable, safer from unwanted pregnancy, infections and abuse. In this way helping young people talk about sexual activities, sexuality and safer sex including the use of condoms. Thus ironing out cultural and religious factors that influence sexual health affecting the status of women and their expectations; and attitude towards young people's sexuality $(4,15,16)$.

In Tanzania, strategies of introducing sex and reproductive education dates back to the mid 1980s. Several induction seminars were run through out the country to teachers who would teach sex and reproductive health education. During the same time Population Family Life Education program (PFL) was running in six regions. Sex and reproductive education is now being debated on its possibility of introducing it in all educational levels. But based on literature surveyed since the idea of introducing sex education in the country no study has been carried out to assess people's acceptability towards introduction of sex and reproductive education in all education levels.

Sex and reproductive health education to adolescents is important now because it is being expected that sex and reproductive health education will empower youths with right information on understanding about their bodies and be responsible of whatever they are doing with their bodies if well designed and taught. The main goal of introducing sex and reproductive health education is to reduce chances of unwanted pregnancies, STIs and HIV infections. The problem, however, is because of the so called 'sensitive in nature' of this subject will it be acceptable to all stakeholders like parents/guardians, religious leaders and the community at large to be introduced at school in all educational levels or to higher classes/grades? If it would be acceptable, how should it be taught at different school levels or on the selected classes/grades? As a separate subject according to sex as was in the past during the initiation period or combined sex as in other subjects? Who should teach the subject matter? Or should it be included in science subjects like in biology and hygiene to reduce sensitivity?

This study aimed at assessing acceptability of parents/guardians towards introduction of sex and reproductive health education at schools in Kinondoni Municipality of Dar es Salaam city as a start by focusing on:-

i. Strategies that are in place for communicating with adolescents on sexuality and reproductive health in urban settings

ii. Assessing acceptability of parents/guardians of adolescents towards introduction of sex and reproductive education in schools

iii. If there is any variation in terms of attitudes towards introduction of sex and reproductive education by religion affiliation

iv. The preferred source of information about sex and reproductive health education to adolescents

The ultimate goal of the study was to get a feedback information on acceptability of the introduction of sex and reproductive health education at primary five to form six (equivalent to grade 5 through grade 14 an age of adolescents) from parents/guardians who are one of the main stakeholders. The results of this study would signal a general reflection of views of Tanzanian population on the possible approaches of introducing sex and reproductive education for improvement of health of adolescents.

\section{Methodology}

A descriptive cross-sectional study involving parents/guardians in the Kinondoni Municipality was conducted between August and September 2004 in Dar es Salaam City. Other municipalities of Dar es Salaam City are Ilala and Temeke. Kinondoni Municipality occupies the northern part of Dar es Salaam city, shares borders with Ilala and Temeke Municipalities. Kinondoni has an area of 531 square kilometres with a population of 1,088, 862 , in which 538,938 (49.9\%) are females (17). Administratively the Municipality has 4 divisions, 27 wards, 14 rural villages and 113 urban hamlets. The indigenous people are Wazaramo and Wamatumbi. However, with increasing urbanisation there are also other ethnic groups from different parts of the country.

Kinondoni Municipality was chosen for this study because it is the largest Municipality in Dar es Salaam City and $43.6 \%$ of the Dar es Salaam City population lives in Kinondoni Municipality. Further, it has many ethnic groups (more than $60 \%$ ) of Tanzania (Tanzania has more than 120 ethnic groups), and thus was expected that chances of getting a representation from different ethnic groups was greater compared to the other Municipalities. Sex and reproductive health education has not been introduced in this Municipality, however most people are informed through mass media.

The study population was parents/guardians with male or female adolescents. This population was deliberately selected because they are likely to be facing challenges on issues of sex and reproductive health from their adolescents. A multi-stage random sampling method was 
used to obtain a representative sample population for the study. First, a simple random technique was used to select 4 wards. The names of the 27 wards were written on small piece of paper; and then put into a box where they were shaken vigorously to ensure thorough mixing; and from there four papers were taken out of the box and the names of the selected wards were recorded. Second, for the respondents the lists of the households with adolescents who were in school were obtained from the ward executive officers of the selected wards. Thirty eight respondents from each ward were obtained by using systematic random sampling technique. Third, in order to get additional opinion/views on the introduction of sex and reproductive health education the study wanted members who would be involved in focus group discussion, should question and challenge the debate of introducing sex and reproductive health education in schools. With the help from teachers and ward executive officers from the chosen wards 32 members were selected, eight from each ward and $50 \%$ of them being women. All participants were briefed about the study and its aim and were requested to consent in writing as participants of the study. The ethical clearance was obtained from Muhimbili University of Health and Allied Sciences Ethics Committee.

Two research instruments were used in data collection after a pilot study in one of the wards in Ilala Municipality. These were a standardised Kiswahili questionnaire for sampled parents/guardians and a guided format for focus group discussion.

Data collection was done through survey using research assistants, whereas for the focus group discussions were done by the researchers themselves. The survey was supplemented with four focus group discussions (one from each ward). The collected data were cleaned by scrutinising each filled questionnaire and eliminating uncalled for information. For quantitative data the collected information were entered in the computer and EPI Info software was used to analyse the data. Percentages and other measures were calculated as indicators of parents/guardians acceptability towards sex and reproductive health education. Whereas the data collected from focus group discussions were analysed using social science and anthropological research methods. The analysed information was summarised; and the results are presented below.

\section{Results}

\section{Demographic characteristics of the respondents}

A total of 150 parents with children aged 10-19 years were interviewed of whom $92(61.3 \%)$ were females. Further analysis showed that majority of the respondents were married and few were single and the rest were either widow or divorced (see Table 1). Whereas on the level of education $107(71.3 \%)$ of the respondents had primary education and $12(8 \%)$ were illiterate. The rest had either secondary or college education, and few attended madrassat. Moslem respondents were the most dominant religion $(71.3 \%)$ among all the respondents when compared with Christians. Almost a half of the respondents were either petty business persons $(36.7 \%)$ or housewives $(49.3 \%)$. The rest were either peasants or involved in informal sector (see Table 1).

\section{Initiation to Adulthood}

The analysis from the focus group discussions and from the survey showed that very little was done from the sides of parents/guardians on sex and reproductive health. Some $(20 \%)$ of the parents/guardians referred their adolescents to elders for jando na unyago (initiation to adulthood-jando for male and unyago for female) within Dar es Salaam, particularly the Wamakonde and Wazaramo ethnic groups. Wamakonde and Wazaramo ethnic groups were still tied with the traditional initiation of their adolescents and very colourful ceremonial and with many presents to the candidate when coming out after initiation. Nevertheless there are marked differences especially on the rituals involved in the initiation process because of different cultural background. Other parents /guardians (10\%) sent their adolescents to respective villages for that purpose because jando na unyago cannot be performed in urban settings.

During the jando na unyago and kufunda mwali (training given to girls focusing on what she is supposed to do in marriage) adolescents were taught on sex and reproductive health; and there were special personnel known in their respective communities for such exercise. The training was done in secret places and nobody was allowed to hear or see what was being done in such places. It was rarely done in urban places and some respondents reported that its absence make the whole process of educating youth cumbersome; increase of social misconduct among the youths. One of the respondents who was an elderly man and illiterate but with respect in the focus group had this to say,

"Jando na unyago teach good manners and that is why we are still surviving with our traditions but our sons and daughters are rude, they cannot hear what we are telling them."

The analysis of the findings showed very few (4\%) of the respondents who did not communicated anything with their adolescents about sexual and reproductive health issues with reason that they were too busy or did not know what to tell them.

\section{Attitude Towards Introduction of Sex and Reproductive Health Education}

In assessing parents/guardians attitude towards introduction of sex and reproductive health education, a total of 5 items on sex and reproductive health issues were used namely; it is important for parents/guardians to talk to their adolescents about sexual issues; use of condoms can protect against STIs and HIV/AIDS; use of the condoms will encourage promiscuity; talking to adolescents about sex and reproductive health education is against their culture; and sex and reproductive health education in school contributes to new STIs and HIV infections. Respondents were asked to either strongly agree or 
strongly disagree on listed items and multiple responses were allowed. The responses are presented in Table 2 .

Table 1: Social characteristics of the study population

\begin{tabular}{lr}
\hline Characteristics & \multicolumn{1}{c}{$\%$} \\
\hline Marital Status & \\
\hline Single & $8.6 \%(13)$ \\
Married & $76.0 \%(114)$ \\
Widowed & $12.6 \%(19)$ \\
Divorced & $2.8 \%(4)$ \\
Total & $\mathbf{1 0 0 \% ( 1 5 0 )}$ \\
Level of education & \\
Primary & $71.3 \%(107)$ \\
Secondary & $12.7 \%(19)$ \\
College & $4.7 \%(7)$ \\
Madras & $3.3 \%(5)$ \\
No formal education & $8.0 \%(12)$ \\
Total & $\mathbf{1 0 0 . 0 \% ( 1 5 0 )}$ \\
Occupation & \\
Peasant & $3.3 \%(5)$ \\
Housewife & $49.3 \%(74)$ \\
Petty business & $36.7 \%(55)$ \\
Business & $2.0 \%(3)$ \\
Employed & $8.7 \%(13)$ \\
Religion & \\
Roman Catholic & \\
Protestant & $20.0 \%(30)$ \\
Moslem & $8.7 \%(13)$ \\
\hline Total & $71.3 \%(107)$ \\
\hline
\end{tabular}

Table 2 Attitude Towards sex education and reproductive health in percentages

\begin{tabular}{lll}
\hline Item used to assess & Strongly agree & $\begin{array}{c}\text { Strongly } \\
\text { disagree }\end{array}$ \\
\hline $\begin{array}{l}\text { 1. It is important for parents to talk to their } \\
\text { adolescence about sexual education and } \\
\text { reproductive health }\end{array}$ & $\mathbf{8 8 . 7 \% ( 1 3 3 )}$ & $11.3 \%(17)$ \\
2. Use of condoms can protect against STI and & $\mathbf{8 2} \%(123)$ & $18 \%(27)$ \\
$\quad$ HIV/AIDS & $78 \%(177)$ & $22 \%(33)$ \\
$\begin{array}{l}\text { 3. Use of condoms will encourage promiscuity } \\
\text { 4. Talking to adolescents about sex education is } \\
\quad \text { against their culture }\end{array}$ & $76.7 \%(115)$ & $\mathbf{2 3 . 3} \%(45)$ \\
$\begin{array}{l}\text { 5. Sex and reproductive health education in } \\
\text { school contributes to new STIs and HIV } \\
\text { infection }\end{array}$ & $\mathbf{5 6 . 0 \% ( 8 4 )}$ & $\mathbf{4 4 . 0 \% ( 6 6 )}$ \\
\hline
\end{tabular}

The analysis of findings in Table 2 presents attitudes toward sex and reproductive health education. More than $80 \%$ of the responses strongly agreed that parents/guardians should talk with adolescents about sex and reproductive health issues and use of condoms could protect against STIs and HIV/AIDS. In contrast more than $70 \%$ strongly agreed that use of condoms would encourage promiscuity and talking to adolescents about sex and reproductive health issues was against their culture; and more that $50 \%$ strongly agreed that sex and reproductive health education would likely contribute to new STIs and HIV/AIDS infections (See Table 2). These finding were underscored by one of the respondents who was a teacher and Muslim in focus group discussion who reported,

"it gives them courage of doing it, after all, since the introduction of condoms, AIDS has been increasing; no relief at all is felt",

Moreover, similar feelings were reflected when the data were analysed according to parents/guardians religious affiliation. All respondents from the major religions (Muslim and Christian) at Kinondoni Municipality were against use of condoms as a measure of prevention. Additionally during the focus group discussion it was learnt that majority of the participants believed that students did practice what they were taught.

During focus group discussion the study also explored the attitude of the participants towards introduction of sex education and reproductive health in schools. The analysis of the study showed that more than half of the female respondents were in favour of introducing sex and reproductive health education and that did not contribute to new STI/HIV infection among adolescents. In contrast more than half of male respondents were not in favour of introduction of sex and reproductive health education.

\section{Variation of views of respondents by religion affiliation towards sex education and reproductive health}

The study explored if there was a variation of views towards introduction of sex and reproductive health education from participants of different religion affiliations i.e the Moslems and Christians (Christian involved Roman Catholic and Protestant). The analysis of the findings showed that more than a half were in favour of the introduction of sex and reproductive health education (about 64\%) with Roman Catholic leading (70\%) followed by Protestants (64\%), and the Moslems (57\%). Nevertheless among the participants and mostly Moslems appeared to be hesitant $(43 \%)$ on the introduction of sex and reproductive health education (See Table 3 ). In addition, the analysis showed that they were traditional cultural restrictions to parents/guardians on communicating sex education and reproductive health issues which was reported to be a problem till to day in some communities of Tanzania. One of the respondents who was a Makonde by ethnic group stressed that point and was echoed by all members of the focus group discussion in that session

"Talking to adolescents about sex and reproductive health education an issue by everybody is against our culture. It is a responsibility of the selected people in the community during the initiation period to adulthood, like that of 'Jando na unyago' and 'kufunda mwali' It is here where sex and reproductive health education to adolescents can take place".

Moreover, during focus group discussion it was learnt there were camps which were meeting places of adolescents in all Dar es Salaam Municipalities and acted as recreation centres to most adolescents. These camps were popular in all Municipalities of Dar es Salaam and sometimes were referred to as vijiweni. It is in these vijiweni where rap songs and poetic drama liked by the adolescents and youths were formulated and practiced. Some of the vijiweni have produced national stars on rap and poetic drama. Nevertheless these vijiweni were highly condemned by parents/guardians because they were claimed to teach bad manners and their members were involved in many high-risk behaviours such as alcoholism, drug abuse and unprotected sexual behaviours.

\section{Preferred source of information on sex education}

The analysis in Table 4 reveals that source of information on sex education and reproductive health education should be from parents/guardians, religious leaders, relatives, peers and others (See Table 3). Methods 
to be used in communicating with adolescents on sex and reproductive health education should include advice $(60.8 \%)$, but others argued that advising a child was like giving him/her authority that was beyond his/her thinking on what was good and bad for him/her. Other methods mentioned were restriction and discussing the pro and cons on various issues related to reproductive health.

Table 3. Attitudes towards sex education by religion in (\%) percentages

\begin{tabular}{llc}
\hline Type of religion & Positive attitude & Negative Attitude \\
\hline Roman Catholic & $70 \%(21)$ & $30.0 \%(9)$ \\
Protestants & $64 \%(8)$ & $36.0 \%(5)$ \\
Moslems & $57 \%(61)$ & $43.0 \%(46)$ \\
Average & $63.7(90)$ & $36.3 \%(60)$ \\
\hline
\end{tabular}

Table 4 Preferred Sources of Information by parents/guardians in percent on Sexual education

\begin{tabular}{lc}
\hline $\begin{array}{l}\text { Who should be the source of } \\
\text { information }\end{array}$ & $\begin{array}{l}\text { \%o of the preferred source of } \\
\text { information }\end{array}$ \\
Parents/guardians & $86.0 \%(129)$ \\
Religious leaders & $70.0 \%(105)$ \\
Media & $62.0 \%(93)$ \\
Health worker & $61.0 \%(91)$ \\
Schools & $59.0 \%(88)$ \\
Relatives & $30.6 \%(46)$ \\
Peers & $14.0 \%(21)$ \\
Others & $4.0 \%(6)$ \\
\hline
\end{tabular}

In the focus group discussion on the other hand, in two groups were on the opinion of slotting sex and reproductive health education in science subject especially in biology and hygiene and partly in social studies. However, they raised doubts on the methods to be used to teach, since it needed a person who could take into account the cultural and religious issues as they relate to reproductive health.

\section{Discussion}

Initiation period to adulthood in the past played the role of educating the adolescents on sexuality and reproductive health through special rituals context that was meant to cement the process. The adolescent period is difficult for most youths like facing peer pressures to take sexual risk by visiting commercial sexual workers and not using condoms $(1,2,4)$. Thus it needs effective communication with qualified personnel within the communities $(1,2,7)$. The initiation to adulthood made the adolescents fit in the community according to sex and defined roles. The completion of the initiation rituals introduces a new period and position in life of the young person.

A change in social structures that were involved in initiation to adulthood as is jando na unyago in Tanzania; has led many traditional values and structures related to sexuality and reproductive health be eroded leaving adolescents at the cross roads $(3,13)$. Thus it was expected that with absence of traditional structure that were involved in initiation to adulthood would lead parents/guardians to have positive attitude towards accepting the introduction of sex and reproductive health education in schools.
But the present findings suggest that there are mixed feelings between parents/guardians and religious leaders on acceptability towards the introduction of sex and reproductive health education; the knowledge and skills which are needed now to avoid sexually transmitted infections (STIs) and HIV infections $(4,13)$. For instance, the analysis in items 1 and 2 in Table 3 show that respondents strongly agreed that it was their role to talk to the adolescents about sex and reproductive health (88.6\%); and use of condoms could protect against STIs and HIV/AIDS (82\%). In contrast to items 3, 4 and 5 the findings suggest that respondents were against sex and reproductive education like the use of condoms encourages promiscuity (78\%) (see Table 3). Further, even though parents/guardians supported strongly that it was important for the parents/guardians to talk to their adolescents about sexual and reproductive health issues, traditional cultures appeared to be inhibiting factor $(76.7 \%)$, and that sex and reproductive health education in schools would likely contribute to new STIs and HIV/AIDS infections (56\%). However, literature review revealed that sex and reproductive health education does not encourage young people to be promiscuous, but helps young people to remain healthy (4).

Moreover, the above analysis of the findings seems to question who should teach and be the source of information on sex and reproductive health education to adolescents in schools. For example, the respondents have shown that the parents /guardians should be the providers of education in this subject matter. This aspect has also been reflected on the preferred sources of information on sex and reproductive education to their adolescents. Parents/guardians ranked first, followed by religious leaders and media ranking third. Health workers and teachers who have access to adolescents ranked fourth and fifth respectively. The present findings seem to suggest that teachers who were targeted to this program were not the right choice. Thus there is a need to include parents/guardians and religious people in designing and implementing the sex and reproductive health education programmes. However during the discussion there were silent on what class sex and reproductive health education should be introduced as well as the need of separating the adolescents according to sex when teaching the subject.

Despite the erosion of traditional culture with increasing modernisation, there are some traditional cultural remnants which seem to play a role in inhibiting the introduction of sex and reproductive health education in schools. For example, in Table 2 item 4, $76.5 \%$ of the responses showed that they could not talk to their adolescents because it was against their culture. They argued that the parents/guardians should send their adolescents to the traditional social structures like those that were involved in jando na unyago. Muhondwa et al (13) in their study found special elderly women who were trainers of unyago in Temeke Municipality, Dar-es-Salaam and were referred as somo. In that study it was further learnt that there were many somos scattered in Dar-esSalaam City. These findings remind us to include the social structures when designing sex and reproductive health education programmes because it appears they have 
a strong hand on it even in urban settings.

During the introduction of sex and reproductive health education in Tanzania in 1980s, religious leaders were against the programme because it was thought it would perpetuate promiscuity to the adolescents. However, in today's findings despite the variation in beliefs the participants from different religion affiliations were in favour of the introduction of sex and reproductive health education, and the Catholic respondents leading. The Catholics religious leaders have even gone further by providing the sex and reproductive health education in different forms through seminars to youths and also during religion classes (authors participated in some of these seminars). Among the Catholics the providers of this education are the sisters and priests. Also the bishops take part at diocese level especially during advent and lent youth seminars.

The focus from these religious people has been mainly on; awareness on the changes of adolescents bodies and its implication to reproductive health, strong sexual desire during the adolescents and how to control oneself, taking preventive measures to infection from HIV/AIDS and STIs, identifying risky zones and how to avoid and use of health facilities for treatment when infected. They have even come up with a guide book on how to fight against HIV/AIDS titled "Njia Sahihi ya Kupambana na UKIMWI (The correct way of fighting against HIV/AIDS") followed by a quotation from the Bible "I am the way, truth and life" John 14:16. Nevertheless all participants from Moslems and Christians were against the use of condoms as a measure of prevention of HIV/AIDS and STIs.

Literature shows that peers and entertainment media are powerful tools on influencing youths to change their behaviour and can provide models of health behaviour if well prepared in the transition period from adolescents to adulthood $(16,18)$. In this study peer groups from vijiweni have a bad reputation by parents/guardians because of what was being observed in these vijiweni. However youth experts can capitalise on the presence of these vijiweni. These vijiweni are initiated by the youths themselves need to be identified and be areas of focus for imparting sex and reproductive health education through seminars, workshops, initiating income generating activities like drama, rap songs and poetic drama which will be educative to the youths on sexuality and reproductive health education. These Vijiweni have even produced national stars in rap songs and music bands like Joseph Haule, Ally Choki, Juma Nature to mention few. All these can make a difference to the youths' behaviours if implemented tactfully.

\section{Conclusion}

Sex and reproductive health education is important to the youths and is expected to provide the knowledge and skills that are needed now to avoid sexual transmitted infections (STI) and HIV infections $(4,15,16,19)$. Despite the mixed feelings found in this study on attitude towards the introduction of sex and reproductive health education, there are some indications that people will change and accept the programme. Preferred source of information like parents/guardians, key people in the community who are respected on issues of initiation to adulthood and religious leaders should be consulted on the content to be included in sex and reproductive heath education and some should participate in teaching the adolescents on sexuality and reproductive health education. In this way taking into consideration of the cultural and religious issues embedded in sexuality and reproductive health.

The other option sex and reproductive health education could be slotted in science subjects especially in biology and hygiene which cover a wide range of issues on family planning and reproductive health, child health, water and sanitation and energy saving strategies to reduce sensitivity of the subject matter. However, the educators should be well prepared and where necessary resource persons from religious institutions, respectable elders versed in cultural issues related to initiation to adulthood should be involved in designing and implementing the programme to be meaningful to the youths and the community at large (15).

\section{Reference:}

1. Swantz, L.M. Religious and Magical Rites of Bantu Women in Tanzania. M. Phil. Thesis Dar-es-Salaam. 1966

2. Swantz L.M Women in Development. A creative role denied. C. Hurts and company, London St. Martin Press, New York, 1985.

3. Kayombo, E. J. and I. Semali, Attitude and Knowledge on Sexuality Among Teenagers and Related Traditional and Cultural Factors. A case of Dar-esSalaam , A consultant report submitted to Centre for the African Studies, Nairobi, 1992

4. Population Information Program Centre for communication, Johns Hopkins School Public Health centre for communication Program (CCP) Population communication Services. Youths in 1980s. Social and health concern, Johns communication Services. Youths in 1980s. Social and health concern, Johns
Hopkins University School of Public Health 111 Baltmore Vol. Xviii no 5 Hopkins University School
November-December 1985.

5. Hauser, G (1973) Longing for Tenderness, Third edition Trobisch Kehl (1973)

6. Wilson, M. Nyakyusa of South Western Tanzania in Molnos, A. (ed) Cultural Sources Material for Population Planning in E. Africa; EA Nairobi, 1973

7. Molnos, A Cultural Source Material for Population Planning in East Africa Vol. III EAP, Nairobi, 1973

8. Mudida, P. F. Development and Promotion of African Traditional medicinal plants and traditional African medicine in the contextual of cultural change: TRAMEDEA experience traditional medicine development Agency (TRAMEDEA) 2002

9. Znaniecki, F. Cultural Sciences. Their origin and Development, Urbana: University of Illinois Press , 1963

10. Wambebe, Charles Guidelines of evaluation of safety and efficacy of African Traditional medicine, International biomedicine Research in Africa (IBRA) Abuja, Nigeria. IDRC Project Number 101915-004.

11. Nicholas D., Ladipo, O.A., Pax J.M. and Otolorin E.O. Sexual behaviour, contraceptive practice, and reproductive health among Nigeria Adolescents. Stud fam plann 1986,17 (2):100-6

12. Mackay, I. Adolescents Fertility, Report of International consultation, Bellagio, London, International Planned Parenthood Federation May 1984.

13. Muhondwa, E. P. ; I.A. Semali.; E. J. Kayombo;.J. M. Bategereza, M. Mwamnyenyelwa, E. Mhina and T. Nyamhanga, A proposed model for community based Prevention of Mother to child transmission of HIV/AIDS, School of Public Health and Social Science, Dar-es-Salaam, Consultant Report $8^{\text {th }}$ September 2004

14. Mbunda, W. M. Adolescent fertility in Tanzania: Knowledge Perception and Practice (Survey Report)Dar-es-Salaam: UMATI, Evaluation and Research Unit 1987

15. United Nations. Joint united Nation Programme on HIV/AIDS (UNAIDS) Impact of HIV and sexual health education on sexual behaviour of young people. Geneva UNAIDS, 1999.

16. Population Information Program Centre for communication, Johns Hopkins School Public Health centre for communication Program (CCP) Population communication Services. Reaching young people world wide. Lesson learned from communication projects 1986-1995Youths in 1980s. Social and Health concern, Johns Hopkins University School of Public Health 111 Baltmore Vol. Xvii no 2 July 1999.

17. United Republic of Tanzania (2002) 2000 housing population census Report 2002

18. Foster, G.M. Traditional Societies and Technological Change. 2nd Edition Harper and Row San Francisco 1973.

19. United Republic of Tanzania Ministry of Health, Tanzania mainland, National AIDS Control Programme. HIV/AIDS/STI Surveillance Report Number 16, January-December 2001.

Received 15 May 2007; Revised 12 February 2008; Accepted 25 February 2008 